\title{
Los centros especializados de gestión de conocimiento: una estrategia de desarrollo desde las regiones
}

\author{
Luis F. Maldonado Granados ${ }^{1}$ \\ Natalia Correa Hincapié \\ Milena Alcócer Tocora ${ }^{3}$
}

\begin{abstract}
Resumen
El trabajo del cual se ocupa este documento se desarrolló con el fin de proponer una estrategia de gestión de conocimiento para potenciar las unidades regionales como espacios de desarrollo autónomo. En una primera parte se presenta una revisión del estado del arte sobre el tema de gestión de conocimiento y desarrollo regional. A partir de este primer estudio se elabora un modelo de centro que integra siete procesos como soporte de sostenibilidad. En la tercera parte se hace un estudio de factibilidad en tres fases: encuestas a líderes administrativos y académicos de la UNAD, entrevistas a expertos y encuentro de propuestas de CGCE. La valoración, tanto de los académicos como de los expertos, concuerda en que el modelo es un aporte conceptual y metodológico para el desarrollo regional. Investigadores de diferentes grupos, especialidades y regiones contribuyen mostrando escenarios de posibilidad para este modelo.
\end{abstract}

Palabras clave: gestión del conocimiento, redes, triple hélice, visibilidad, prospectiva, gestión informática.

1 Programa de doctorado en Gestión de conocimiento de la Universidad Nacional Abierta y a Distancia; investigador del Grupo TECNICE.

2 Docente de la Escuela de Ciencias Agrícolas, Pecuarias y del Medio Ambiente; investigadora del Grupo de Investigación y Desarrollo Agroambiental.

3 Docente de la Escuela de Ciencias Básicas, Tecnología e Ingeniería de la Universidad Nacional Abierta y a Distancia; investigadora del Grupo GIEPRONAL. 


\title{
Knowledge management centers: a region- based development strategy
}

\begin{abstract}
The work with which this document deals was developed in order to propose a knowledge management strategy to enhance regional units as spaces for autonomous development. A first part presents a review of state of the art on the theme of regional development and knowledge management. From this first study, we develop a model for a center with seven processes as support of sustainability. On the third part we conduct a study of feasibility in three phases: polls to administrative and academic leaders of the UNAD, interviews with experts and a meeting about CGCE proposals. Valuation of academics and experts agreed that the model is a conceptual and methodological contribution to regional development. Researchers from different groups, specialties and regions contribute showing possible scenarios for this model.
\end{abstract}

Key words: knowledge management, networks, triple helix, visibility, forecasting, information management.

Recibido: 16 de septiembre de 2010 Aceptado: 29 de octubre de 2010

\section{Introducción}

Una explicación válida de la falta de equidad social se puede dar desde la perspectiva cognitiva, y una estrategia de acción inteligente para superarla, en términos contemporáneos, parte de la gestión de conocimiento. Stehr (1999) analiza cómo las fuerzas de estratificación social vienen evolucionando en la sociedad del conocimiento. A diferencia de épocas anteriores, el conocimiento predomina sobre la disposición de riquezas materiales como posesión de tierras y equipos. El conocimiento se constituye como conjunto de capacidades que permiten a las personas dar valor agregado al uso de las cosas y defenderse de los riesgos inherentes a la actividad laboral, la economía y la política. Las formas de organización familiar y de agremiación han sufrido cambios y giran alrededor del conocimiento como base de la actividad productiva.

La iniciativa de centros de gestión de conocimiento especializado se presenta como un dispositivo orientado a que las comunidades se integren y avancen en la sociedad de conocimiento. Es una forma de empoderamiento vinculada a temas 
centrales de su actividad productiva y una estrategia orientada a generar desarrollo con equidad social. Siguiendo a Stehr (1999), se puede ver como un instrumento que afecta la manera en que las comunidades adquieren, disponen y preservan los instrumentos tradicionales de estratificación como la tierra y otros instrumentos de producción.

El escenario de la empresa en la sociedad del conocimiento se interpreta siguiendo el principio expresado por Garrat (1990), según el cual las empresas necesitan aprender por lo menos al ritmo del cambio del entorno para poder sobrevivir en el mundo de hoy. Las diferencias en el desarrollo de las regiones se profundizan en la medida en que este principio tiene menos vigencia en los sitios más apartados de los centros de investigación y desarrollo científico, tecnológico y de innovación.

\section{Antecedentes}

\section{La dinámica de la construcción de conocimiento en las organizaciones}

El modelo de Nonaka y Takeuchi (1995) otorga a los modelos mentales un papel fundamental en la gestión del conocimiento y avanza en el estudio de su formación. El conocimiento nuevo se crea a partir de la interacción entre conocimiento tácito y explícito. Esto lleva a postular cuatro formas de transformación del conocimiento (ilustración 1). El conocimiento puede pasar de tácito en una persona a tácito en otra, a lo que llaman socialización. Puede estar tácito en una persona y hacerlo explícito, lo que configura una externalización. Una persona lo puede hacer explícito y comunicarlo a otra persona que a su vez muestra conocimiento explícito, lo que se denomina combinación. Cuando se pasa de conocimiento explícito en una persona a otra que lo torna tácito, se denomina internalización.

La socialización inicia con la construcción de un "campo" de interacción que facilita compartir experiencias y modelos mentales entre los miembros de un grupo o comunidad. La externalización se activa por el diálogo o la reflexión del colectivo. En la externalización ayudan las metáforas y analogías para articular la representación del conocimiento tácito con ayuda de representaciones previas. La combinación es activada por la formación de redes sociales, al traer componentes de conocimiento de diferentes miembros del colectivo o de fuentes externas para construir el nuevo conocimiento. Finalmente, la práctica (aprender haciendo) habilita la internalización.

Jonassenand \& Henning (1996) distinguen tres clases de modelos relacionados con el conocimiento: a) el modelo mental es la estructura de categorías de valores que representan el mundo, es el resultado de la interacción con el entorno, se actualiza permanentemente y determina nuestro comportamiento, actitudes y decisiones; b) los modelos conceptuales se usan en representaciones explícitas 
de eventos, sistemas y procesos, en formas tales como textos, videos o discursos orales; c) los modelos cognitivos son formas de describir los modelos mentales y los procesos de conocimiento, y son utilizados por los científicos de la cognición.

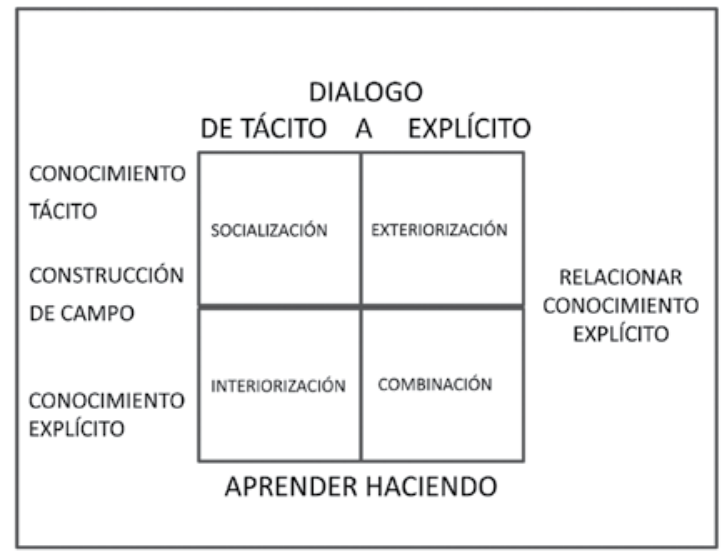

Ilustración 1. Modelo de Nonaka y Takeuchi (1995). La dinámica de construcción de conocimiento.

Los modelos mentales son representaciones internas del mundo. Su mejor indicador es el lenguaje, aunque también aparecen en el comportamiento y el desempeño en tareas. Una buena representación la constituyen las redes de conceptos. Una condición válida para su observación e identificación es la solución de problemas pertinentes.

Para Martindale (1993), el modelo mental es la base de la predicción de futuro, sin la cual los procesos de adaptación y control del entorno serían imposibles. Este enfoque constituye una base cognitiva en la introducción de ejercicios de análisis prospectivo como mecanismo de interiorización del conocimiento científico.

Los conocimientos previos constituyen sin lugar a duda la base del significado. El entorno da contenido experiencial a la representación. Por esta razón, la gestión de conocimiento debe tomar en cuenta estos parámetros para ser exitosa.

\section{La gestión de conocimiento desde la perspectiva de la convergencia digital}

La convergencia digital lleva a interpretar el desarrollo contemporáneo en términos de la integración de tecnologías de la comunicación a la gestión de conocimiento, la representación del mundo y la comunicación entre las diferentes formas de organización de los actores. Por este motivo, el concepto de brecha digital aparece en la agenda de los organismos interesados en la equidad social y económica. El carácter de empoderamiento se expresa al afirmar que cuando las tecnologías de 
la información son usadas por las personas, les permiten desarrollar iniciativas que de otra manera no serían posibles (Mansell, 2002).

\section{El desarrollo regional y la gestión de conocimiento}

Cooke \& Leydesdorff (2006) presentan el concepto de región con base en la relación gobierno y área geográfica. Relacionan el término latino regio con el término regere, que significa gobernar una división administrativa de un país. Otras relaciones están asociadas como las culturales, económicas y de vecindad.

Las relaciones de vecindad generan fenómenos de influencia mutua de unidades regionales en el desarrollo $\mathrm{y}$, en particular, en el desarrollo de conocimiento vinculado a la producción. Si una región A genera un desarrollo de conocimiento, es muy probable que la región vecina $B$ tenga un efecto de diseminación de conocimiento. Los modelos de economía basada en conocimiento superan el modelo tradicional que atribuye el desarrollo económico a la integración de tierra-trabajo-capital. Surgen en una perspectiva sistémica al analizar la calidad de los factores de producción. La calidad del conocimiento de la fuerza laboral se analiza en relación con otros factores, por la necesidad de generar políticas nacionales de desarrollo económico. Los organismos de planeación se ven motivados a construir mapas de conocimiento para proyectar el desarrollo. Esta visión sistémica lleva a percibir desequilibrios entre las regiones en producción, niveles de vida y gestión de conocimiento. El desarrollo de redes de comunicación hace pensar en niveles de integración de las regiones para un desarrollo sostenido de los sistemas nacionales o internacionales.

Una forma de ver las diferencias en desarrollo de las regiones ha tomado en consideración la ventaja competitiva de un entorno, como lo hace David Ricardo. Por ejemplo, el clima y el suelo propicio para producir vino generan ventajas en la comercialización del producto. Una interpretación reciente consideraba el costo de mano de obra como factor de ventaja competitiva. Sin embargo, el desarrollo tecnológico en entornos sin ventaja competitiva natural ha mostrado que la ventaja competitiva se puede generar en la medida en que se integra conocimiento a un ramo de la actividad económica. Esto da lugar al concepto de ventaja construida.

La ventaja construida requiere la integración de acciones del campo de la economía, el gobierno, la infraestructura de conocimiento, la cultura y la comunidad. La infraestructura de ventaja construida habilita la acción de triple hélice: gobiernoindustria-universidad (Leydesdorff,1994). Se focaliza en la relación de tres componentes de la economía basada en conocimiento: centro de producción de conocimiento, el mercado y el gobierno. La universidad es dinamizadora de los procesos de innovación incubando empresas, gestionando monitoreo de la producción y el mercado y habilitando la formación de competencias. Un ejemplo de triple hélice fue desarrollado por el Instituto Tecnológico de Massachusetts (Etzkowitz, 2002). 
Leydesdorff (2006) amplía el concepto de triple hélice a una estrategia acorde con el modo 2 de producción de conocimiento. La evolución de la teoría sobre el desarrollo ha pasado por diferentes etapas. En un momento se miró la formación de recursos humanos como capital humano y capital intelectual. Otra tendencia introdujo el concepto de economía del conocimiento señalando el conocimiento como un recurso comercializable tal como se planteó en el proyecto Quinta Generación de los japoneses. Posteriormente se viró a la economía basada en el conocimiento para señalar que el valor agregado de la producción está basado en el conocimiento. Luego el plan nacional de innovación se postula como el factor integrador de componentes múltiples de una economía nacional. Más recientemente la economía se flexibiliza como resultado de los procesos de comunicación, dando lugar al concepto de globalización. La innovación se convierte en un proceso en permanente construcción dirigido por las expectativas codificadas y no tanto por determinantes históricos. Es aquí donde el concepto de prospectiva logra ocupar un lugar de importancia.

El conocimiento se valida a través de la comunicación en contexto y ésta puede habilitar dinámicas de cambio en las comunidades. La comunicación codificada se diferencia y focaliza. Al hacerlo tiende a formar círculos con cierta autonomía $\mathrm{y}$, para habilitar la integración, requiere procesos de interfaz entre los diferentes actores y escenarios. Es el fenómeno que se puede presentar entre los productores, los académicos y los políticos. Los primeros desarrollan su discurso alrededor de la producción de riqueza, los segundos codifican procesos de innovación mediante ciencia y tecnología, y los terceros desarrollan sus códigos en relación con el control. El concepto de unidad regional y la integración con base en conceptos como los de cadenas productivas adquieren importancia y se convierten en estrategia para entender y actuar en un sistema de alta complejidad.

Los escenarios de gestión de conocimiento han sido típicamente urbanos. Pocos estudios se muestran aplicados a las regiones rurales. Morales (2007) describe un proceso desarrollado en una comunidad rural de Baja California con el enfoque de región de aprendizaje. La iniciativa surge con la llegada de dos personas que, interesadas en cultivos orgánicos en 1985, forman inicialmente una organización de seis personas. Ésta se propuso producir de acuerdo con los estándares internacionales y alrededor de esta meta giró la formación de una comunidad de aprendizaje para buscar continuamente la mejor forma de lograr sus objetivos. Un año después había 18 personas vinculadas y cuatro años más tarde, más de 160 con una producción superior al millón de dólares. La organización fue diferenciando funciones e integrando redes de intercambio. Los gobiernos local y regional apoyaron la iniciativa que se convirtió en movimiento. El aprendizaje se consolidó a partir de problemas concretos. Así fueron acumulando conocimiento sobre el control de plagas, la producción de abono orgánico, las formas más eficientes de empaque, comercialización y contratación. El aprendizaje se consolidó de forma solidaria. Los agricultores eran dueños de su propia producción, pero tenían muchas actividades en forma colaborativa. El gobierno actuó para consolidar 
la confianza mutua y facilitar el intercambio con universidades y centros de investigación. La organización se vinculó a la dinámica global y pudo probar los beneficios para sus miembros y sus familias.

\section{Clusters de conocimiento}

Scheel (2002) señala que uno de los factores de riqueza y prosperidad en los países desarrollados es la existencia de sistemas sostenibles capaces de convertir las innovaciones tecnológicas en productividad industrial, riqueza y productividad global, pero que poco se ha capitalizado de esta experiencia para impulsar el desarrollo de los países menos desarrollados. Propone el modelo llamado de las cinco 1 (linking, leverage, learning, leadership, and alignment) dirigido a la administración de redes colaborativas de diferentes organizaciones para un propósito común: el desarrollo económico, social, político y cultural de las regiones en desarrollo.

El primer componente consiste en vincular las organizaciones (academia, banco, gobierno e industria) en un solo sistema enfocado en un mismo fin. El segundo componente se enfoca en encontrar las competencias requeridas para que los actores puedan actuar con eficiencia en un contexto mundial. El tercer componente organiza un sistema de aprendizaje con componentes como la observación de empresas exitosas (benchmarking), organización de información, experiencias piloto, incubadoras de empresas. El cuarto componente consiste en adecuación tecnológica para ser eficientes. El quinto componente apunta a liderar innovación para resolver los problemas y retos que se visualizan en un contexto global y que requieren una organización de liderazgo.

Clasifica los retos de la infraestructura en: soporte financiero, programas gubernamentales de apoyo, programas de investigación y desarrollo tecnológico, programas de formación en innovación, programas de gestión tecnológica, programas de consultoría, programas de infraestructura física y programas de infraestructura de conocimiento como bibliotecas virtuales y repositorios.

\section{Las redes sociales y su relevancia en la gestión del conocimiento}

El trabajo de Mischen y Jackson (2008) enfoca el desarrollo regional integrando tres perspectivas: la gestión de conocimiento, la teoría de la complejidad y las redes sociales. Siguiendo el pensamiento de la teoría evolucionaria de los sistemas complejos, los sistemas sociales integran unidades pequeñas en sistemas superiores que ganan en autonomía (Holland, 1995). Iniciativas como los CGCE serían organizaciones complejas cuyo vínculo integrador es el conocimiento aplicado al desarrollo de las actividades fundamentales de la producción y del desarrollo humano. La metodología de análisis de redes sociales muestra una forma de visualizar este desarrollo. En este enfoque, para que una organización logre sus objetivos requiere entender: a) la dinámica de la red social en su interior 
y de su organización como miembro de una red de organizaciones; b) las rutinas que surgen como patrones de comportamiento; c) los elementos fundamentales que le dan fortaleza en el logro de sus propósitos.

Si bien las organizaciones locales o regionales valoran las fortalezas de sus espacios, sus comunidades tienen el riesgo de aislar su pensamiento de los contextos más amplios. Esta observación es notoria en relación con la gestión del conocimiento. Si se desea controlar este riesgo, puede ser una estrategia valiosa actuar desde las representaciones de conocimiento vinculando actores en sus escenarios geográficos y mecanismos de comunicación. En esta dirección, Liebowitz (2005) desarrolla el concepto de mapa de conocimiento como el proceso que relaciona tópicos, personas, documentos, ideas, y relaciones con fuentes externas, con sus respectivas densidades y la manera como se pueden consultar. Para la construcción de mapas de conocimiento el autor sugiere el uso de la metodología de análisis de redes sociales para ver la fuerza de las relaciones entre actores ubicados en sus espacios, y la metodología de análisis de procesos jerárquicos (Saaty, 1980) para valorar la necesidad del conocimiento en relación con la toma de decisiones. El enfoque de estos dos métodos permite integrar la visión de necesidad con la de construcción de tejido social.

En el consenso de la comunidad científica actual, las redes de conocimiento son una posibilidad para producir, intercambiar $\mathrm{y}$, de alguna manera, transferir el conocimiento que se produce o se aprende dentro de un contexto global o específico. Los estudios empíricos muestran que los vínculos sociales son de crucial importancia para la generación de ideas creativas, innovadoras y para el conocimiento de diferentes actividades asociadas con las comunidades que hacen parte de la red (Fliaster A., Spiess J., 2008).

Según Yulianow et al. ( 2003), las redes de conocimiento se establecen entre individuos, grupos y organizaciones y valoran la integridad de sus actividades. Casas (2001) incluye en este concepto las redes profesionales y de entrenamiento, y las de difusión y transmisión de conocimientos o de innovaciones, que aportan a la formación de espacios regionales de conocimiento.

En un contexto sociológico y dinámico, Royero (2008) concibe las redes sociales de conocimiento como interacciones humanas en la producción, almacenamiento, distribución, transferencia, acceso y análisis de conocimientos producidos de manera sistemática (investigación). En estas redes hay motivación por compartir datos de cualquier índole y a través de cualquier medio, especialmente electrónico, y normalmente buscan comprender fenómenos y procesos así como transformar su entorno.

Las redes sociales de conocimiento implican tanto una relación directa con la tecnología como la relación humana que permite el crecimiento integral de sus miembros. En la dimensión tecnológica, la plataforma de comunicación es un 
dispositivo que posibilita la gestión integral de interacción. En la dimensión humana la red tiene capacidad para transformar el entorno, que es de enriquecimiento intelectual constante, y para crear mediante la investigación científica, sistemática y multidisciplinaria.

Concepto y modelo metodológico de un centro de gestión de conocimiento especializado

La revisión de antecedentes nos lleva a analizar la gestión de conocimiento para el desarrollo de las regiones como un proceso eminentemente cognitivo entre actores que tienen escenarios de acción y aprendizaje muy diferentes. Por una parte están los científicos y académicos que actúan en escenarios de investigación, generalmente urbanos, y que interactúan con base en ontologías y lenguajes especializados. Por otra parte están los actores de las zonas rurales que interactúan usando lenguajes y conceptos surgidos de la práctica empírica, en cadenas de producción gobernadas por leyes de comercio, normalmente encubiertas para ellos. La gestión del conocimiento para el desarrollo debe llegar a integrar estos dos polos generando cambios en los procesos cognitivos y en los patrones de acción o cultura de los colectivos y asegurar mejoramiento cualitativo de procesos vitales.

\section{Los procesos de intercambio cognitivo en la gestión de conocimiento}

Todos los seres vivos aprenden integrando su herencia genética con los procesos cognitivos que se activan como resultado de la experiencia. Más aun, los conocimientos que se adquieren van modificando la estructura cognitiva de tal manera que los nuevos conocimientos se integran a conocimientos previos. La relación entre actores es un proceso comunicativo donde cada actor afecta el nivel cognitivo del otro. El sistema de conceptos de los actores interactúa en procesos de comunicación que dan como resultado sistemas ontológicos compartidos.

En consecuencia, los centros actúan con el principio del reconocimiento de los saberes de cada actor, luego con la activación de la comunicación de esos procesos y con la codificación o representación de ese conocimiento, que sirve de insumo para la construcción conjunta de nuevas representaciones.

El centro de gestión de conocimiento especializado toma un ámbito de conocimiento con significado vital para una comunidad en sentido económico y social. Por ejemplo, en una comunidad dedicada a la explotación del banano, un centro de gestión de conocimiento sobre esta fruta tiene plena legitimidad.

\section{El modelo de centro de gestión de conocimiento especializado (CGCE)}

A continuación se propone un modelo de gestión de conocimiento basado en procesos como base para la consulta a actores expertos en gestión de conocimiento. 


\section{Construcción del campo - objeto de conocimiento:}

El campo de acción está constituido por un sector de la actividad económica que postula como objeto de gestión de conocimiento. Ejemplos de estos campos son: la producción de lácteos, la producción de fruta en conserva, la producción de mango, la producción de verdura y hortaliza, la explotación racional de los recursos hídricos, etc. El objeto de conocimiento se analiza desde diferentes perspectivas: económica, científica, técnica, social, de sostenibilidad, etc.

\section{Construcción de una comunidad de innovación:}

Es el primer paso en la construcción de un CGCE. Está constituida por un equipo gestor del que hacen parte un investigador de la unad, investigadores y estudiantes de semilleros de investigación. En un segundo paso ingresan miembros de una comunidad con dedicación a la actividad productiva en un campo - objeto de conocimiento. En tercer lugar se vinculan otros actores de los sectores comercial, político y educativo.

\section{El ejercicio de la observación de procesos:}

En una etapa siguiente se introduce la observación activa de los procesos de producción en sus diferentes dimensiones. Esta información se sistematiza en forma de estructura de monitoreo y se integra al conocimiento disponible para interpretar el estado actual del sistema de producción.

\section{Integración informática de los centros:}

Los dispositivos informáticos como son computadores conectados a bases de datos usando internet, se organizan de tal manera que puedan cumplir funciones como las siguientes: consulta, sistematización de la información de monitoreo de procesos, uso de software para hacer modelamiento y análisis prospectivo.

\section{Ejercicio de prospectiva a partir de la integración de saberes:}

En esta etapa se tiene como objetivo el reconocimiento de los saberes de los participantes y del conocimiento disponible en forma documental y en expertos que puedan interactuar con la comunidad. En segundo lugar se persigue formar una metodología que permita construir conjuntamente escenarios prospectivos a partir del reconocimiento de conocimientos actuales del colectivo. Es consistente con la posición según la cual la innovación es un proceso en permanente construcción dirigido por las expectativas codificadas y no tanto por determinantes históricos (Leydesdorff, 2006). 
6. Gestión de proyectos para mejorar la producción de riqueza y de conocimiento:

A partir de la construcción de escenarios en el análisis prospectivo, se diseñan proyectos. Éstos se orientan a generar nuevo conocimiento e introducir innovaciones a partir del conocimiento integrado, convirtiéndose en objeto de gestión para obtener recursos y hacerlos viables. Se hace seguimiento a su desarrollo y se implementan estrategias para la sostenibilidad de las innovaciones generadas.

\section{Visibilidad de los centros:}

Un elemento importante del sistema de información es que soporte la visibilidad del CGCE. Sus actores con sus producciones se hacen visibles, de tal manera que otras comunidades puedan entrar en contacto con el colectivo que es el CGCE y, a su vez, éste entre en contacto y colaboración con otras organizaciones. El sistema debe desarrollar un sistema de aula abierta con repositorio de objetos de aprendizaje que resultan del proceso de construcción de conocimiento.

\section{Consolidación de redes sociales como mecanismo para formar cultura:}

El colectivo se diseña y se gestiona con metodología de análisis de redes sociales. De esta manera se monitorea su evolución estructural. La red tiene un método de formación diseñado. En este diseño se encuentran tanto la producción de conocimiento propio como la de expertos e investigadores y las experiencias de comunidades del mismo campo.

\section{Análisis del modelo y resultados}

La validación del modelo propuesto se hace en tres etapas: 1) análisis prospectivo mediante encuesta a académicos de diferentes regiones de Colombia; 2) entrevista a expertos de varios países; 3 ) encuentro de investigadores de regiones diferentes de Colombia.

\section{Aplicación de cuestionario a académicos:}

El cuestionario se aplicó a 33 directivos, 24 académicos, cinco administrativos, dos egresados y tres estudiantes de diferentes programas de la UNAD, en el marco del seminario de liderazgo desarrollado por la Institución. La Tabla 1 muestra la proveniencia de los encuestados. La respuesta fue voluntaria.

El ordenamiento de mayor a menor según la probabilidad de que se den los eventos identificados en los ocho procesos es la siguiente: Construcción de comunidad de innovación: 6.10, ejercicio de prospectiva: 5.96, construcción de campo: 5.44, 


\section{Luis F. Maldonado, Natalia Correa y Milena Alcócer}

Los centros especializados de gestión de conocimiento: una estrategia de desarrollo desde las regiones, artículo producto de la investigación

gestión de proyectos: 5.44, observación de procesos: 4.86, visibilidad de los centros: 4.85, integración informática: 4.80 , consolidación de redes sociales: 4.55 .

Tabla 1. Distribución de los encuestados según la zona geográfica donde trabajan

\section{Zona}

No. de encuestas

Amazonía, Orinoquía, (Amazonas, Casanare,

Meta, Guaviare, Guainía, Arauca )

Bogotá-Cundinamarca

19

Boyacá

Caribe (Magdalena, Guajira, Cesar, Córdoba,

8

9

Bolívar, Atlántico, Sucre)

Oriente (Santander, Norte de Santander)

Occidente (Antioquia, Quindío, Risaralda, Caldas,

9

Chocó)

Centro Sur (Nariño, Valle, Cauca)

4

Sur (Caquetá, Huila, Tolima)

Total

67

\section{Estadisticas por evento}

Tabla 2. Estadística descriptiva de las respuestas por escenario y por proceso

\begin{tabular}{|l|l|l|l|l|l|}
\hline Escenario & Proceso & Media & Rango & $\begin{array}{l}\text { Desviación } \\
\text { estándar }\end{array}$ & $\begin{array}{l}\text { Media por } \\
\text { proceso }\end{array}$ \\
\hline $\begin{array}{l}\text { A1. La comunidad } \\
\text { convierte su actividad } \\
\text { económica en objeto de } \\
\text { conocimiento y estudio }\end{array}$ & $\begin{array}{l}\text { Construcción de } \\
\text { campo }\end{array}$ & 5,38 & $0-10$ & 2,31 & \\
\hline $\begin{array}{l}\text { A2. La comunidad } \\
\text { estudia su actividad } \\
\text { económica desde } \\
\text { diferentes perspectivas } \\
\text { (científica, económica, } \\
\text { técnica, social, etc.). }\end{array}$ & $\begin{array}{l}\text { Construcción de } \\
\text { campo }\end{array}$ & 5,50 & $0-10$ & 2,33 & 5,44 \\
\hline
\end{tabular}




\begin{tabular}{|c|c|c|c|c|c|}
\hline Escenario & Proceso & Media & Rango & $\begin{array}{l}\text { Desviación } \\
\text { estándar }\end{array}$ & $\begin{array}{l}\text { Media por } \\
\text { proceso }\end{array}$ \\
\hline $\begin{array}{l}\text { A3. Se integra } \\
\text { una comunidad de } \\
\text { innovación constituida } \\
\text { por investigadores, } \\
\text { estudiantes } \\
\text { universitarios miembros } \\
\text { de semilleros de } \\
\text { investigación. }\end{array}$ & $\begin{array}{l}\text { Construcción de } \\
\text { comunidad de } \\
\text { innovación }\end{array}$ & 6,49 & $0-10$ & 2,33 & \\
\hline $\begin{array}{l}\text { A4. Se integra una } \\
\text { comunidad de inno- } \\
\text { vación constituida por } \\
\text { investigadores de la } \\
\text { universidad, estudiantes } \\
\text { de semilleros de inves- } \\
\text { tigación, productores de } \\
\text { un sector, comerciantes } \\
\text { y miembros del sector } \\
\text { político. }\end{array}$ & $\begin{array}{l}\text { Construcción de } \\
\text { comunidad de } \\
\text { innovación }\end{array}$ & 5,72 & $0-10$ & 2,43 & 6,10 \\
\hline $\begin{array}{l}\text { A5. La comunidad } \\
\text { monitorea su sistema de } \\
\text { producción. }\end{array}$ & $\begin{array}{l}\text { Observación de } \\
\text { procesos }\end{array}$ & 4,97 & $0-10$ & 2,15 & \\
\hline $\begin{array}{l}\text { A6. La comunidad } \\
\text { introduce un sistema de } \\
\text { observación e informa- } \\
\text { ción de sus procesos } \\
\text { productivos. }\end{array}$ & $\begin{array}{l}\text { Observación de } \\
\text { procesos }\end{array}$ & 4,85 & $0-10$ & 2,17 & \\
\hline $\begin{array}{l}\text { A7. La comunidad } \\
\text { integra un sistema de } \\
\text { información de su acti- } \\
\text { vidad productiva y hace } \\
\text { ejercicios de prospecti- } \\
\text { va con ese sistema de } \\
\text { información }\end{array}$ & $\begin{array}{l}\text { Observación de } \\
\text { procesos }\end{array}$ & 4,94 & $0-10$ & 2,06 & 4,86 \\
\hline $\begin{array}{l}\text { A8. La comunidad usa } \\
\text { software para simular } \\
\text { sus procesos dinámicos } \\
\text { de producción. }\end{array}$ & $\begin{array}{l}\text { Integración infor- } \\
\text { mática }\end{array}$ & 4,06 & $0-10$ & 2,36 & \\
\hline $\begin{array}{l}\text { A9. Las comunidades } \\
\text { organizan internet para } \\
\text { consultar información } \\
\text { especializada sobre sus } \\
\text { procesos productivos. }\end{array}$ & $\begin{array}{l}\text { Integración infor- } \\
\text { mática }\end{array}$ & 5,55 & $0-10$ & 2,65 & 4,80 \\
\hline
\end{tabular}




\begin{tabular}{|c|c|c|c|c|c|}
\hline Escenario & Proceso & Media & Rango & $\begin{array}{l}\text { Desviación } \\
\text { estándar }\end{array}$ & $\begin{array}{l}\text { Media por } \\
\text { proceso }\end{array}$ \\
\hline $\begin{array}{l}\text { A10. Un miembro de } \\
\text { la comunidad socializa } \\
\text { su conocimiento sobre } \\
\text { su actividad productiva } \\
\text { ante los demás miem- } \\
\text { bros de su comunidad. }\end{array}$ & $\begin{array}{l}\text { Ejercicio de pros- } \\
\text { pectiva }\end{array}$ & 5,62 & $0-9$ & 2,35 & \\
\hline $\begin{array}{l}\text { A11. Un miembro de la } \\
\text { comunidad reconoce } \\
\text { el conocimiento de los } \\
\text { otros miembros de la } \\
\text { comunidad. }\end{array}$ & $\begin{array}{l}\text { Ejercicio de pros- } \\
\text { pectiva }\end{array}$ & 5,82 & $0-10$ & 2,30 & \\
\hline $\begin{array}{l}\text { A12. El experto identi- } \\
\text { fica el conocimiento de } \\
\text { la comunidad sobre su } \\
\text { campo de experticia. }\end{array}$ & $\begin{array}{l}\text { Ejercicio de pros- } \\
\text { pectiva }\end{array}$ & 6,65 & $0-10$ & 1,96 & \\
\hline $\begin{array}{l}\text { A13. La comunidad } \\
\text { construye escenarios } \\
\text { prospectivos en interac- } \\
\text { ción con un experto. }\end{array}$ & $\begin{array}{l}\text { Ejercicio de pros- } \\
\text { pectiva }\end{array}$ & 5,75 & $0-10$ & 2,20 & 5,96 \\
\hline $\begin{array}{l}\text { A14. La comunidad } \\
\text { genera proyectos de } \\
\text { innovación y desarrollo } \\
\text { a partir de ejercicios de } \\
\text { visión prospectiva. }\end{array}$ & $\begin{array}{l}\text { Gestión de pro- } \\
\text { yectos }\end{array}$ & 5,10 & $0-10$ & 2,31 & \\
\hline $\begin{array}{l}\text { A15. La comunidad } \\
\text { gestiona recursos para } \\
\text { desarrollar proyectos de } \\
\text { innovación y desarrollo }\end{array}$ & $\begin{array}{l}\text { Gestión de pro- } \\
\text { yectos }\end{array}$ & 5,71 & $0-10$ & 2,25 & \\
\hline $\begin{array}{l}\text { A16. La innovación } \\
\text { exitosa hace que los } \\
\text { proyectos desarrollados } \\
\text { sean sostenibles. }\end{array}$ & $\begin{array}{l}\text { Gestión de pro- } \\
\text { yectos }\end{array}$ & 5,50 & $0-10$ & 2,37 & 5,44 \\
\hline $\begin{array}{l}\text { A17. La comunidad } \\
\text { hace visible su cono- } \\
\text { cimiento sobre su pro- } \\
\text { ducción para que otras } \\
\text { comunidades entren en } \\
\text { contacto con ellas. }\end{array}$ & $\begin{array}{l}\text { Visibilidad de los } \\
\text { centros }\end{array}$ & 5,44 & $0-10$ & 2,17 & \\
\hline
\end{tabular}




\begin{tabular}{|c|c|c|c|c|c|}
\hline Escenario & Proceso & Media & Rango & $\begin{array}{l}\text { Desviación } \\
\text { estándar }\end{array}$ & $\begin{array}{l}\text { Media por } \\
\text { proceso }\end{array}$ \\
\hline $\begin{array}{l}\text { A18. La comunidad pro- } \\
\text { duce objetos virtuales } \\
\text { para hacer visible su } \\
\text { conocimiento. }\end{array}$ & $\begin{array}{l}\text { Visibilidad de los } \\
\text { centros }\end{array}$ & 3,80 & $0-9$ & 2,18 & \\
\hline $\begin{array}{l}\text { A19. La comunidad con- } \\
\text { sulta el conocimiento de } \\
\text { otras comunidades. }\end{array}$ & $\begin{array}{l}\text { Visibilidad de los } \\
\text { centros }\end{array}$ & 5,22 & $0-10$ & 2,08 & 4,85 \\
\hline $\begin{array}{l}\text { A20. La comunidad usa } \\
\text { metodología de análisis } \\
\text { de redes sociales para } \\
\text { evaluar su integración. }\end{array}$ & $\begin{array}{l}\text { Consolidación de } \\
\text { redes sociales }\end{array}$ & 4,36 & $0-10$ & 2,36 & \\
\hline $\begin{array}{l}\text { A21. La comunidad } \\
\text { tiene un sistema de } \\
\text { formación permanente } \\
\text { que integra su propio } \\
\text { conocimiento con el } \\
\text { de expertos y de otras } \\
\text { comunidades afines. }\end{array}$ & $\begin{array}{l}\text { Consolidación de } \\
\text { redes sociales }\end{array}$ & 4,73 & $0-8$ & 1,99 & 4,55 \\
\hline Media eventos & & 5,29 & & 1,56 & \\
\hline
\end{tabular}

En la valoración de los escenarios, la probabilidad asociada es mayor para los procesos relacionados con la innovación y menor para los procesos de control y visibilidad (observación, sistematización y consolidación de redes). La desviación estándar es relativamente alta en todos los casos, pues es mayor a dos puntos y el rango de variación es también grande. Esto significa que el acuerdo entre los participantes es notorio (tabla 2).

En la segunda parte de la encuesta, las hipótesis con mayor valor corresponden a la estructura tipo triple hélice. En general, los datos son similares y tienen menos variaciones relativas (valor de las desviaciones estándar y los rangos de las respuestas) comparados con la valoración de eventos (tabla 3).

Tabla 3. Valoración de hipótesis por parte de los encuestados

\begin{tabular}{|l|l|l|l|}
\hline Hipótesis & Media & Rango & $\begin{array}{l}\text { Desviación } \\
\text { estándar }\end{array}$ \\
\hline $\begin{array}{l}\text { H1. Si una comunidad toma como objeto de estudio } \\
\text { su actividad productiva, entonces sus miembros } \\
\text { integran nuevo conocimiento a esa actividad. }\end{array}$ & 7,96 & $2-10$ & 2,05 \\
\hline
\end{tabular}




\begin{tabular}{|c|c|c|c|}
\hline Hipótesis & Media & Rango & $\begin{array}{l}\text { Desviación } \\
\text { estándar }\end{array}$ \\
\hline $\begin{array}{l}\text { H2. Si las comunidades convierten su actividad } \\
\text { económica en objeto de conocimiento y lo analizan } \\
\text { desde diferentes perspectivas, generan innovaciones } \\
\text { sostenibles. }\end{array}$ & 8,14 & $2-10$ & 1,94 \\
\hline $\begin{array}{l}\text { H3. Si se constituye una comunidad de innovación } \\
\text { integrada por expertos de la unad, semilleros de } \\
\text { investigación y miembros de una comunidad eco- } \\
\text { nómica, entonces se potencia simultáneamente la } \\
\text { producción económica y la producción de conoci- } \\
\text { miento científico. }\end{array}$ & 8,64 & $4-10$ & 1,7 \\
\hline $\begin{array}{l}\text { H4. Si se construyen escenarios prospectivos } \\
\text { conjuntamente entre expertos y miembros de una } \\
\text { comunidad productiva, entonces se integra la ciencia } \\
\text { a la cultura de la comunidad. }\end{array}$ & 8,38 & $3-10$ & 1,91 \\
\hline $\begin{array}{l}\text { H5. Si en la actividad productiva se integran los } \\
\text { ambientes digitales a la simulación, monitoreo y } \\
\text { formación, entonces la inequidad basada en la distri- } \\
\text { bución de conocimiento se supera. }\end{array}$ & 7,4 & $2-10$ & 2,24 \\
\hline $\begin{array}{l}\text { H6. Si la comunidad genera proyectos de innovación } \\
\text { a partir de ejercicios de visión prospectiva, entonces } \\
\text { mejora la eficiencia en la gestión de recursos. }\end{array}$ & 8,1 & $1-10$ & 2 \\
\hline $\begin{array}{l}\text { H7. Si la comunidad hace visible su producción de } \\
\text { conocimiento, entonces potencia su dinámica de } \\
\text { cambio. }\end{array}$ & 8,46 & $4-10$ & 1,72 \\
\hline $\begin{array}{l}\text { H8. Si una comunidad introduce mecanismos de } \\
\text { monitoreo de su estructura como red social, mejora } \\
\text { su cohesión y capacidad de acción integrada. }\end{array}$ & 8,47 & $4-10$ & 1,59 \\
\hline MEDIA & 8,17 & & 1,44 \\
\hline
\end{tabular}

\section{Análisis de entrevistas a expertos}

Se realizaron ocho entrevistas a expertos en el campo de la prospectiva, la ciencia y la tecnología provenientes de Colombia, Argentina, Venezuela, Chile, México, Brasil, Alemania y España. 
Tabla 4. Comentario de los expertos sobre la estructura del CGCE

\begin{tabular}{|c|c|}
\hline ASPECTOS & COMENTARIOS \\
\hline Estructura de los CGCE & $\begin{array}{l}\text { - Es importante tener un sistema de seguimiento } \\
\text { nacional e internacional de la actividad econó- } \\
\text { mica. } \\
\text { - Las organizaciones y sobre todo esta organiza- } \\
\text { ción de interacción deben intentar empezar. } \\
\text { - Se debe aplicar una metodología prospectiva ya } \\
\text { que hasta ahora la propuesta se ha hecho dentro } \\
\text { de un marco lógico. } \\
\text { - La actividad económica no se puede ver sola- } \\
\text { mente desde la mirada del desarrollo económico } \\
\text { (mirada sistémica). } \\
\text { - Se debe incorporar a microcomunidades porque } \\
\text { debe haber sostenibilidad de los procesos. } \\
\text { - Las organizaciones inteligentes se pueden fre- } \\
\text { nar por lo afectivo. Debe haber tejido social para } \\
\text { hacer inclusión. Es necesaria la disponibilidad. } \\
\text { - Se sugiere que el punto de construcción de } \\
\text { redes sociales esté dentro de la formación de la } \\
\text { comunidad de innovación. } \\
\text { - Es importante caracterizar los diferentes roles, } \\
\text { especificar el flujo de inputs y outputs para } \\
\text { cada rol y entre ellos, actores, roles, intereses y } \\
\text { objetivos. }\end{array}$ \\
\hline Procedimiento de CGCE & $\begin{array}{l}\text { - Se debe hacer una caracterización que permita } \\
\text { hacer una aproximación antropológica y psicoló- } \\
\text { gica para motivar la participación. } \\
\text { - Es importante hacer sentir a la comunidad que } \\
\text { lo que se está haciendo tiene sentido. } \\
\text { - Se piensa en enfocarlo primero al incremento } \\
\text { de la producción. } \\
\text { - Se sugiere revisar en detalle el tema de canali- } \\
\text { zación de recursos. } \\
\text { - Se deben generar estrategias para que la } \\
\text { comunidad científica y el gobierno validen el } \\
\text { conocimiento producido y brinden apoyo. } \\
\text { - La prospectiva puede orientar la conformación } \\
\text { de la comunidad de innovación. }\end{array}$ \\
\hline
\end{tabular}




\begin{tabular}{|l|l|}
\hline ASPECTOS & COMENTARIOS \\
\hline Experiencias similares & $\begin{array}{l}\text { - Expresan que es novedoso y demasiado } \\
\text { ambicioso como está propuesto. }\end{array}$ \\
& $\begin{array}{l}\text { - Se manifiesta que no se ha visto un ejercicio } \\
\text { pareado que integre los componentes propuestos } \\
\text { y agregan que han visto modelos con los } \\
\text { componentes pero de forma separada. } \\
\text { - Se tienen referencias de experiencias } \\
\text { presentadas por observatorios y se opina } \\
\text { que a nivel latinoamericano hacen gestión de } \\
\text { conocimiento. } \\
\text { - En Latinoamérica se está trabajando en } \\
\text { modelos de intervención: seguridad, droga, } \\
\text { delincuencia común, inclusión de género, } \\
\text { básicamente ONG. No se sabe de universidades } \\
\text { que estén trabajando en este campo. }\end{array}$ \\
\end{tabular}

Los expertos estuvieron de acuerdo en que la estrategia permitirá llegar a la resolución de problemas como la inequidad. Sin embargo, plantean que es importante definir claramente el objeto de conocimiento o actividad económica, los actores y sus metodologías de articulación para garantizar el éxito.

La tabla 4 muestra los comentarios más relevantes de los expertos frente al análisis de la estructura, la dinámica y la existencia de la iniciativa en otros países.

Las observaciones realizadas por los expertos permiten argumentar nuevamente la pertinencia y la viabilidad que tiene la propuesta para aportar al desarrollo de los encuentros de investigadores.

Se desarrolló un encuentro de investigadores provenientes de varias zonas del país, enfocado al análisis de la propuesta inicial y a la discusión sobre la relación Universidad-Empresa-Estado. Cada investigador, desde su perspectiva, muestra la factibilidad de este tipo de centros. Los investigadores muestran a los CGCE como una propuesta viable y como un espacio que permite la contextualización de los procesos académicos para hacer innovaciones reales. Ellos destacan las características de una institución como la unad, con presencia en diferentes regiones del contexto nacional, para ampliar el espectro de posibilidades de investigación, acompañadas de programas avanzados de formación. Los CGCE pueden aportar al desarrollo y a la equidad nacional, al generar una nueva visión de progreso que integrará a la sociedad en sus diferentes ámbitos. La Tabla 5 muestra 16 opciones de CGCE. 
Tabla 5. Posibles campos de estudio para CGCE, presentados por los investigadores de diferentes zonas de Colombia

\begin{tabular}{|c|c|c|}
\hline CGCE & Necesidad & Factibilidad \\
\hline $\begin{array}{l}\text { Name. } \\
\text { Carlos Ramón Vidal Tovar, } \\
\text { CEAD Simón Bolívar en } \\
\text { Cartagena }\end{array}$ & $\begin{array}{l}\text { Importante en Brasil, Colombia, } \\
\text { Haití, Venezuela y Antillas } \\
\text { francesas. En Colombia la } \\
\text { zona costera del departamento } \\
\text { de Córdoba, la región natural } \\
\text { de los montes de María en } \\
\text { los departamentos de Sucre y } \\
\text { Bolívar, y algunos municipios de } \\
\text { los departamentos del Cesar y } \\
\text { la Guajira. }\end{array}$ & $\begin{array}{l}\text { Su explotación se realiza } \\
\text { en predios de economía } \\
\text { campesina con bajo nivel de } \\
\text { tecnificación. Se presenta } \\
\text { como una oportunidad para } \\
\text { generar investigaciones } \\
\text { multidisciplinarias que } \\
\text { beneficien a la comunidad } \\
\text { de agricultores, estudiantes, } \\
\text { profesionales y empresas } \\
\text { interesadas. }\end{array}$ \\
\hline $\begin{array}{l}\text { Reproducción animal } \\
\text { Ruth Amanda Acero Camelo del } \\
\text { CEAD de Facatativá }\end{array}$ & $\begin{array}{l}\text { Las enfermedades } \\
\text { reproductivas se diseminan en } \\
\text { los animales de importancia } \\
\text { zootécnica. Los sistemas } \\
\text { tradicionales introducen nuevos } \\
\text { sistemas que ofrecen ventajas } \\
\text { comparativas importantes. }\end{array}$ & $\begin{array}{l}\text { El conocimiento y las tecno- } \\
\text { logías que se han generado } \\
\text { en este campo hacen que los } \\
\text { avances reproductivos en las } \\
\text { explotaciones pecuarias hayan } \\
\text { cambiado sus sistemas tradicio- } \\
\text { nales y se hayan encaminado } \\
\text { hacia unos nuevos que les } \\
\text { ofrecen ventajas comparativas } \\
\text { importantes. Los presupuestos } \\
\text { de investigación y su aplicación } \\
\text { tienden a incrementarse. }\end{array}$ \\
\hline $\begin{array}{l}\text { Biodiversidad. Adriana } \\
\text { Alexandra Maldonado } \\
\text { Chaparro, CEAD José Celestino } \\
\text { Mutis en Bogotá }\end{array}$ & $\begin{array}{l}\text { A. Profundizar e incrementar el } \\
\text { conocimiento de la diversidad } \\
\text { biológica de nuestro país. } \\
\text { B. Proponer estrategias de ma- } \\
\text { nejo in situ y ex situ que garanti- } \\
\text { cen la sostenibilidad económica } \\
\text { de los recursos biológicos. } \\
\text { C. Coordinar y ejecutar progra- } \\
\text { mas y estrategias de manejo } \\
\text { para la conservación, el ma- } \\
\text { nejo, la recuperación y el uso } \\
\text { sostenible de la biodiversidad. } \\
\text { D. Contribuir al conocimiento } \\
\text { popular sobre valores y usos } \\
\text { sostenibles de la diversidad } \\
\text { biológica. }\end{array}$ & $\begin{array}{l}\text { Se proyecta como en } \\
\text { un centro de calidad en } \\
\text { investigaciones relacionadas } \\
\text { con la conservación, } \\
\text { uso y aprovechamiento } \\
\text { sostenible de los recursos } \\
\text { biológicos del país. Realizará } \\
\text { estudios especializados } \\
\text { e interdisciplinarios que } \\
\text { contribuirán al desarrollo } \\
\text { científico, cultural, económico } \\
\text { y social de país, así como al } \\
\text { establecimiento de estrategias } \\
\text { que procuren el uso sostenible } \\
\text { de nuestros recursos biológicos. }\end{array}$ \\
\hline
\end{tabular}


Luis F. Maldonado, Natalia Correa y Milena Alcócer

Los centros especializados de gestión de conocimiento: una estrategia de desarrollo desde las regiones, artículo producto de la investigación

\begin{tabular}{|c|c|c|}
\hline CGCE & Necesidad & Factibilidad \\
\hline $\begin{array}{l}\text { Productos naturales no } \\
\text { maderables de la Amazonia y } \\
\text { Orinoquía colombianas. } \\
\text { Luz Mery Bernal, Gustavo } \\
\text { Forero, CEAD José Celestino } \\
\text { Mutis }\end{array}$ & $\begin{array}{l}\text { La región de la Orinoquia } \\
\text { priorizó, a través de los planes } \\
\text { regionales de competitividad } \\
\text { coordinados por el Ministerio de } \\
\text { Comercio, Industria y Turismo, } \\
\text { los productos que considera } \\
\text { promisorios, atendiendo a su } \\
\text { interés comercial, cosmético, } \\
\text { farmacéutico, alimenticio y } \\
\text { artesanal. Se busca mejorar el } \\
\text { bienestar de las comunidades } \\
\text { a través de oportunidades } \\
\text { laborales, transformación } \\
\text { y comercialización de los } \\
\text { productos y aseguramiento de } \\
\text { la continuidad de las especies. } \\
\text { Lo anterior fue propuesto por } \\
\text { las comisiones regionales } \\
\text { de competitividad de los } \\
\text { departamentos de Casanare, } \\
\text { Guainía, Vichada y Guaviare. }\end{array}$ & $\begin{array}{l}\text { El centro estará orientado hacia } \\
\text { la producción, transformación, } \\
\text { comercialización y consumo } \\
\text { de productos no maderables } \\
\text { de la Amazonia y Orinoquía } \\
\text { colombiana como: marañón } \\
\text { (Anacardium occidentale), } \\
\text { ají (Capsicum frutescens), } \\
\text { chiqui-chiqui sacha inchi } \\
\text { (Plukenetia volubilis), arazá } \\
\text { (Eugenia stipitata), asaí } \\
\text { (Euterpe oleracea), seje } \\
\text { (Jessenia Polycarpia,) e inchi } \\
\text { (Caryodendron orinocense). }\end{array}$ \\
\hline $\begin{array}{l}\text { Centro de Conocimiento } \\
\text { Ancestral y Tradicional. } \\
\text { Gin Rusbel T. Puerto Nariño, } \\
\text { Amazonas }\end{array}$ & $\begin{array}{l}\text { Se necesita configurar la } \\
\text { conciencia histórica y la } \\
\text { identidad autónoma y colectiva } \\
\text { de los pueblos indígenas } \\
\text { del Amazonas colombiano } \\
\text { a través del estudio de las } \\
\text { actividades etnoculturales } \\
\text { desde los diferentes ámbitos, } \\
\text { proyectándolos hacia un } \\
\text { desarrollo social sostenible y } \\
\text { sustentable. }\end{array}$ & $\begin{array}{l}\text { El centro pretende fortalecer } \\
\text { los conocimientos ancestrales } \\
\text { y tradicionales de las culturas } \\
\text { pertenecientes a las etnias } \\
\text { Ticuna, Cocama y Yagua. } \\
\text { Igualmente se pretende generar } \\
\text { espacios de diálogo intercultural } \\
\text { promovidos desde la iniciativa } \\
\text { local hacia los niveles } \\
\text { nacionales e internacionales. }\end{array}$ \\
\hline
\end{tabular}




\begin{tabular}{|c|c|c|}
\hline CGCE & Necesidad & Factibilidad \\
\hline $\begin{array}{l}\text { Sancha inchi. Manuel Tangoa } \\
\text { Farías, Puerto Nariño }\end{array}$ & $\begin{array}{l}\text { Para el municipio de Puerto } \\
\text { Nariño, Amazonas, su principal } \\
\text { unidad de producción es } \\
\text { la chagra, y actividades } \\
\text { extractivas como la pesca, la } \\
\text { caza y la recolección de frutos. } \\
\text { En el municipio se encuentran } \\
\text { chagras que cultivan maíz, } \\
\text { yuca, plátano, caña y frutales } \\
\text { que son utilizados para el } \\
\text { consumo, y los excedentes son } \\
\text { comercializados en la cabecera } \\
\text { municipal. La costumbre de } \\
\text { actividades extractivas ha } \\
\text { ocasionado el bajo desarrollo } \\
\text { tecnológico en la región y } \\
\text { la baja implementación de } \\
\text { proyectos productivos, lo que } \\
\text { tarde o temprano provocará un } \\
\text { agotamiento del recurso. }\end{array}$ & $\begin{array}{l}\text { El centro es una estrategia } \\
\text { para que los investigadores } \\
\text { de la unad encuentren nuevas } \\
\text { alternativas de producción que } \\
\text { contribuyan con el desarrollo de } \\
\text { la región y mejoren la calidad } \\
\text { de vida de sus habitantes. }\end{array}$ \\
\hline $\begin{array}{l}\text { Sistemas de alimentación para } \\
\text { rumiantes. } \\
\text { Ruth Amanda Acero Camelo }\end{array}$ & $\begin{array}{l}\text { Cerca del } 70 \% \text { de los costos } \\
\text { de producción corresponden a } \\
\text { la alimentación en los sistemas } \\
\text { de producción animal. Hay } \\
\text { dependencia de materias } \\
\text { primas importadas y algunas } \\
\text { compiten con la alimentación } \\
\text { humana (soya, maíz). Hay } \\
\text { un aporte significativo de la } \\
\text { ganadería a las emisiones de } \\
\text { metano. Hay subproductos } \\
\text { de la Industria con potencial } \\
\text { de uso. El pequeño productor } \\
\text { dispone de sistemas de } \\
\text { alimentación incipientes. }\end{array}$ & $\begin{array}{l}\text { El centro estaría orientado al } \\
\text { monitoreo de las siguientes } \\
\text { variables: Componentes } \\
\text { actuales de los sistemas de } \\
\text { alimentación; fluctuación de } \\
\text { precios de componentes de la } \\
\text { dieta; costos de alimentación } \\
\text { animal, incluyendo forraje; } \\
\text { promedios de producción de } \\
\text { leche; contenidos de nutrientes } \\
\text { en la leche, ganancias diarias } \\
\text { de peso, conversiones } \\
\text { alimenticias; intervalo entre } \\
\text { partos; pesos y edad al } \\
\text { primer servicio; servicios por } \\
\text { concepción efectiva y peso } \\
\text { al nacimiento; precios del } \\
\text { producto final, fluctuaciones } \\
\text { en el año y rentabilidad de la } \\
\text { producción. }\end{array}$ \\
\hline
\end{tabular}


Luis F. Maldonado, Natalia Correa y Milena Alcócer

Los centros especializados de gestión de conocimiento: una estrategia de desarrollo desde las regiones, artículo producto de la investigación

\begin{tabular}{|c|c|c|}
\hline CGCE & Necesidad & Factibilidad \\
\hline $\begin{array}{l}\text { Tabaco. Germán Garcés, CEAD } \\
\text { de Málaga, Santander }\end{array}$ & $\begin{array}{l}\text { El sector tabacalero de la } \\
\text { región de Málaga, Santander, } \\
\text { es altamente vulnerable, de } \\
\text { características etnográficas } \\
\text { y productivas específicas, } \\
\text { endémicas, evolutivas de } \\
\text { lo tradicional a modelos } \\
\text { adaptativos de desarrollo. Se } \\
\text { necesita un seguimiento a la } \\
\text { estructura socioeconómica y } \\
\text { cultural, que plasme y evalúe } \\
\text { posibles alternativas para el } \\
\text { desarrollo tabacalero regional } \\
\text { e identifique condiciones reales } \\
\text { de producción, comercialización } \\
\text { y sostenibilidad de los } \\
\text { tabacaleros. }\end{array}$ & $\begin{array}{l}\text { EI CGCE haría prospección } \\
\text { tecnológica para indicar } \\
\text { oportunidades y amenazas } \\
\text { al desarrollo tecnológico y } \\
\text { señalaría las demandas para } \\
\text { las tecnologías. Analizaría el } \\
\text { funcionamiento de los sistemas } \\
\text { sociales y económicos donde la } \\
\text { tecnología va a funcionar. Con } \\
\text { base en una visión holística, } \\
\text { desarrollaría una prospección } \\
\text { tecnológica. }\end{array}$ \\
\hline $\begin{array}{l}\text { Arracacha. Milena Alcócer } \\
\text { Tocora }\end{array}$ & $\begin{array}{l}\text { En el corregimiento de } \\
\text { Anaime se están adelantando } \\
\text { innovaciones tecnológicas a } \\
\text { partir de la obtención de harina } \\
\text { y su transformación en arequipe } \\
\text { y torta de arracacha, por parte } \\
\text { de un grupo de mujeres. Esta } \\
\text { iniciativa minimiza las pérdidas } \\
\text { generadas en la cosecha del } \\
\text { producto que no reúne las } \\
\text { condiciones para mercados } \\
\text { de Bogotá y Cali, los cuales } \\
\text { compran el } 80 \% \text { y } 10 \% \text { de } \\
\text { la producción diaria. Bogotá } \\
\text { consume } 38750 \text { Kg (38.75 } \\
\text { Ton.) y los mercados de Cali e } \\
\text { lbagué, } 14250 \text { Kg (14.25 Ton.). } \\
\text { La producción ha disminuido } \\
\text { en } 10 \% \text { en los dos últimos años } \\
\text { debido a fluctuación en precios } \\
\text { y demanda inelástica, típica de } \\
\text { producto agrícola, y a manejos } \\
\text { de post cosecha y fitosanitarios } \\
\text { inadecuados. }\end{array}$ & $\begin{array}{l}\text { El centro aprovecharía las } \\
\text { potencialidades en productos } \\
\text { agroindustriales derivados } \\
\text { de la arracacha. La relativa } \\
\text { baja inversión y el mercado } \\
\text { potencial internacional abren } \\
\text { oportunidades. Por otra parte, la } \\
\text { comunidad ya tiene una cultura } \\
\text { y una actitud positiva vinculada } \\
\text { a esta actividad económica. }\end{array}$ \\
\hline
\end{tabular}




\begin{tabular}{|c|c|c|}
\hline CGCE & Necesidad & Factibilidad \\
\hline $\begin{array}{l}\text { Apicultura. Ricardo Tello, CEAD } \\
\text { de Ibagué }\end{array}$ & $\begin{array}{l}\text { El departamento del Tolima es } \\
\text { una gran despensa agrícola. } \\
\text { En los últimos años ha } \\
\text { encaminado esfuerzos para } \\
\text { desarrollar la fruticultura por la } \\
\text { oferta de suelos, clima y vías } \\
\text { de comunicación, lo que hace } \\
\text { que esta región tenga ventajas } \\
\text { competitivas y comparativas } \\
\text { para el desarrollo de este } \\
\text { tipo de cultivos. Las zonas } \\
\text { sembradas en la actualidad y } \\
\text { las nuevas áreas adecuadas } \\
\text { con riego en el proyecto } \\
\text { "Triángulo del sur del Tolima” } \\
\text { van a constituir el polo de } \\
\text { desarrollo frutícola del país. }\end{array}$ & $\begin{array}{l}\text { Buscando competitividad en } \\
\text { la producción se requiere } \\
\text { la utilización de las abejas } \\
\text { como agentes polinizadores, } \\
\text { las cuales de forma natural } \\
\text { incrementan la producción } \\
\text { desde el } 20 \% \text { en el caso de los } \\
\text { cítricos hasta más del } 100 \% \text { en } \\
\text { el caso de cucurbitáceas como } \\
\text { melón, sandia y ahuyama. Esto } \\
\text { hace que los cultivos sean más } \\
\text { rentables y puedan incrementar } \\
\text { el área demandando mano de } \\
\text { obra, lo cual, a su vez, genera } \\
\text { el desarrollo económico de la } \\
\text { región. }\end{array}$ \\
\hline $\begin{array}{l}\text { Piscicultura. Deya Amparo } \\
\text { Pérez, CEAD de Neiva }\end{array}$ & $\begin{array}{l}\text { En los últimos } 20 \text { años la } \\
\text { producción mundial piscícola ha } \\
\text { aumentado significativamente, } \\
\text { destacándose tilapia, trucha } \\
\text { y cachama que han crecido } \\
\text { a ritmos de } 12 \%, 6 \% \text { y } 29 \% \\
\text { respectivamente. (Acuerdo } \\
\text { de competitividad cadena } \\
\text { piscícola, 2005). }\end{array}$ & $\begin{array}{l}\text { El centro focalizaría el estudio } \\
\text { de procesos de globalización y } \\
\text { tendencias del mercado, la po- } \\
\text { sibilidad de internacionalización } \\
\text { (CEAD Florida), la formación de } \\
\text { recursos humanos especializa- } \\
\text { dos en el área. Aprovecharía } \\
\text { las convocatorias de investi- } \\
\text { gación e innovación que se } \\
\text { están abriendo y el avance en } \\
\text { política agraria de apoyo a los } \\
\text { pequeños productores. }\end{array}$ \\
\hline $\begin{array}{l}\text { Desarrollo sostenible y } \\
\text { seguridad alimentaria. William } \\
\text { Montealegre, CEAD Pitalito. }\end{array}$ & $\begin{array}{l}\text { Colombia, un país con } \\
\text { 1'141.748 kilómetros cuadrados } \\
\text { donde habitan } 42.3 \text { millones } \\
\text { de personas, espera para } 2020 \\
\text { una población de } 57 \text { millones y } \\
\text { de } 60 \text { millones para } 2025 .\end{array}$ & $\begin{array}{l}\text { El centro se focalizaría a res- } \\
\text { ponder la pregunta: ¿Cómo me- } \\
\text { jorar las condiciones generales } \\
\text { de la población, entre ellas la } \\
\text { seguridad alimentaria, teniendo } \\
\text { en cuenta aspectos como la } \\
\text { distribución de la propiedad y } \\
\text { del ingreso, la situación polí- } \\
\text { tica y social por la que el país } \\
\text { atraviesa, la institucionalidad, la } \\
\text { sostenibilidad, y, en general, el } \\
\text { equilibrio de la sociedad en su } \\
\text { conjunto? }\end{array}$ \\
\hline
\end{tabular}


Luis F. Maldonado, Natalia Correa y Milena Alcócer

Los centros especializados de gestión de conocimiento: una estrategia de desarrollo desde las regiones, artículo producto de la investigación

\begin{tabular}{|c|c|c|}
\hline CGCE & Necesidad & Factibilidad \\
\hline $\begin{array}{l}\text { Movilidad humana. María del } \\
\text { Pilar Triana, CCAV Pereira }\end{array}$ & $\begin{array}{l}\text { La movilidad humana es un } \\
\text { fenómeno de cambio que se } \\
\text { expande casi incontroladamente } \\
\text { más allá de las fronteras } \\
\text { tradicionales. Dentro de las } \\
\text { categorías sociales se tienen: } \\
\text { migrantes e itinerantes, } \\
\text { emigrantes, foráneos, } \\
\text { transmigrantes, refugiados, } \\
\text { desplazados, traficados, } \\
\text { deportados, retornados, } \\
\text { exiliados, aeronavegantes, } \\
\text { peregrinos, turistas, gitanos, } \\
\text { circenses, recreacionistas y } \\
\text { ambulantes. }\end{array}$ & $\begin{array}{l}\text { El Centro se focalizaría al } \\
\text { monitoreo prospectivo y al } \\
\text { desarrollo de proyectos y } \\
\text { redes sociales que canalicen la } \\
\text { dinámica social y económica del } \\
\text { fenómeno de movilidad social } \\
\text { en regiones específicas. }\end{array}$ \\
\hline $\begin{array}{l}\text { Recursos hídricos. Natalia } \\
\text { Correa, } \\
\text { CEAD de Medellín }\end{array}$ & $\begin{array}{l}\text { Debido a su ubicación } \\
\text { geográfica y a sus condiciones } \\
\text { de relieve, Colombia tiene una } \\
\text { precipitación media anual de } \\
3.000 \mathrm{~mm} \text {, que representa una } \\
\text { riqueza importante de recursos } \\
\text { hídricos, cuando es comparada } \\
\text { con el promedio mundial de } \\
\text { precipitación anual, } 900 \mathrm{~mm}, \\
\text { y con el promedio anual de } \\
\text { Suramérica, del orden de los } \\
1.600 \mathrm{~mm} \text {. En términos del } \\
\text { caudal específico de escorrentía } \\
\text { superficial, Colombia presenta } \\
58 \mathrm{l} / \mathrm{s} / \mathrm{km} 2 \text {, tres veces mayor } \\
\text { que el promedio sudamericano } \\
\text { (21 l/s/ km2) y seis veces mayor } \\
\text { que la oferta hídrica específica } \\
\text { promedio a nivel mundial(10 } \\
\text { l/s/km2). }\end{array}$ & $\begin{array}{l}\text { El centro se orientaría al } \\
\text { desarrollo de proyectos } \\
\text { que mejoren la recolección, } \\
\text { tratamiento y disposición de las } \\
\text { aguas residuales, para evitar } \\
\text { la contaminación ambiental } \\
\text { y sanitaria de las fuentes } \\
\text { abastecedoras de agua y } \\
\text { preservar la disponibilidad del } \\
\text { recurso hídrico. }\end{array}$ \\
\hline
\end{tabular}




\begin{tabular}{|c|c|c|}
\hline CGCE & Necesidad & Factibilidad \\
\hline $\begin{array}{l}\text { Floricultura. Susana Gómez, } \\
\text { Eje cafetero. }\end{array}$ & $\begin{array}{l}\text { En los años 90, la floricultura } \\
\text { se expande desde las grandes } \\
\text { empresas de la Sabana, } \\
\text { especializadas en la producción } \\
\text { de flores tradicionales, hacia } \\
\text { la agricultura de tipo familiar } \\
\text { y pequeñas empresas } \\
\text { productoras de menos de } \\
10 \text { hectáreas, las cuales se } \\
\text { dedican a la producción de } \\
\text { flores no tradicionales y follajes } \\
\text { de forma artesanal, como } \\
\text { alternativa de diversificación } \\
\text { ante la crisis de otros sectores, } \\
\text { especialmente el del café. } \\
\text { La tecnología e investigación } \\
\text { desarrolladas en el país han } \\
\text { girado en torno a las flores } \\
\text { tradicionales como rosa, clavel } \\
\text { y crisantemo. Para el resto } \\
\text { de especies existen grandes } \\
\text { vacíos de conocimiento. }\end{array}$ & $\begin{array}{l}\text { El Centro se orientaría al } \\
\text { fortalecimiento de procesos } \\
\text { asociativos para pequeños } \\
\text { floricultores, encaminados al } \\
\text { mejoramiento de los procesos } \\
\text { productivos y al acopio de } \\
\text { productos que permita obtener } \\
\text { volúmenes en cantidad y } \\
\text { calidad consistente durante } \\
\text { todo el año, para la exportación. } \\
\text { También desarrollaría procesos } \\
\text { de investigación participativa } \\
\text { y extensión de resultados; } \\
\text { daría asistencia técnica } \\
\text { a pequeños productores } \\
\text { desde la práctica profesional } \\
\text { dirigida y contribuiría a la } \\
\text { formación de los trabajadores } \\
\text { de explotaciones florícolas } \\
\text { encaminados a obtener la } \\
\text { certificación en competencias } \\
\text { laborales. }\end{array}$ \\
\hline Bosques & $\begin{array}{l}\text { La problemática básica en } \\
\text { bosques está determinada por: } \\
\text { Fragmentación de los territorios, } \\
\text { extracción de productos del } \\
\text { bosque, pérdida de suelos, } \\
\text { pérdida de diversidad, bajo } \\
\text { nivel de educación de los } \\
\text { pobladores, mala cobertura de } \\
\text { salud, regulares sistemas de } \\
\text { transporte, malos trabajos y } \\
\text { explotación alta de los recursos } \\
\text { propios, utilización de servicios } \\
\text { ambientales sin ningún } \\
\text { control, y desplazamiento. No } \\
\text { conocemos casos de fugas de } \\
\text { cerebros o de conocimiento. }\end{array}$ & $\begin{array}{l}\text { Los temas de estudio del centro } \\
\text { podrían ser: cambio climático, } \\
\text { captura de carbono, biología } \\
\text { de poblaciones, biología } \\
\text { de comunidades, ecología } \\
\text { del paisaje, restauración, } \\
\text { diversidad, fauna, conocimiento } \\
\text { tradicional, transformación } \\
\text { de productos, aguas, } \\
\text { genética, paleo-ecología, } \\
\text { dendrocronología, migración de } \\
\text { especies animales y vegetales, } \\
\text { aprovechamiento, maderas, } \\
\text { productos no maderables, } \\
\text { turismo, bosque natural, bosque } \\
\text { plantado, certificación forestal, } \\
\text { bosque urbano, silvicultura, } \\
\text { clima urbano. }\end{array}$ \\
\hline
\end{tabular}




\section{Conclusiones}

El estado del arte muestra que los problemas de equidad social en la sociedad del conocimiento surgen de la manera como las organizaciones integran conocimiento a su propio desarrollo, armonizando el ritmo de aprendizaje al ritmo de cambio en el entorno. Los instrumentos tradicionales de estratificación, como pueden ser la tierra y otros instrumentos de producción, son afectados por la capacidad que adquieren las personas para dar valor a los factores de producción.

En el caso particular del desarrollo regional, los desequilibrios se pueden contrarrestar en la medida en que la ubicación geográfica se convierte en espacio de aprendizaje y la distancia es controlada por medio de la integración digital y las redes sociales activas en las cadenas productivas.

El ejercicio que se hace con este trabajo tiene carácter prospectivo. Es una estructura que tiene valor en la medida en que sirve de base para la generación de estructuras de acción concretas. La fuerza de la propuesta la da la integración de los ocho procesos presentados en forma de sistema dinámico. Los diferentes actores involucrados en la construcción de la propuesta de CGCE concuerdan que, con esta base, este tipo de proyectos es viable. Es una racionalidad que asegura la sostenibilidad y la proyección de la iniciativa.

La propuesta conserva el sentido de integración hecho por los teóricos de la triple hélice, ampliada a la manera como lo hacen Leydesdorff (2006) y Scheel (2002). Aquí se explicita el papel de los diferentes sectores de la sociedad cuando se integran en objetivos comunes, con la conciencia de generar aprendizaje en las dinámicas de producción sostenible.

Este enfoque de integración es percibido por los participantes en este trabajo como fundamental para que puedan funcionar las redes sociales que hacen posible la integración del conocimiento científico con el conocimiento derivado de la experiencia de las unidades regionales y aplicarlo al desarrollo de la producción, la generación de competencias de los actores y de la prospectiva que habilite la construcción consciente de futuro.

El trabajo presenta un escenario realista desde la perspectiva de los investigadores, con antecedentes concretos en el desarrollo de CGCE específicos con objetos de conocimiento relativamente elaborados en comunidades integradas y con gran potencial de acción.

La investigación y la innovación son necesarias para la gestión del conocimiento en cualquiera de los objetos de conocimiento identificados regionalmente. El escenario para los investigadores es muy promisorio y, si se consideran las políticas actuales en ciencia y tecnología en Colombia, la iniciativa podría contribuir significativamente al movimiento de investigación modo dos. 
La visibilidad de los centros se traduce en la estrategia de intercambio de conocimiento entre los actores del CGCE y la comunidad en general. La visibilidad asegura la referencia y la valoración positiva en la continuidad de los procesos. La construcción y evolución de los CGCE debe estar ligada al desarrollo prospectivo de los objetos de conocimiento, para que las comunidades usen la identificación de sus propias necesidades como base de construcción de futuros mejores.

Finalmente queremos presentar la idea de CGCE como una estrategia para la creación tanto de académicos como de personas vinculadas a la producción, al sector financiero y al sector político y que están interesadas en contribuir a la superación de problemas de equidad social.

\section{Referencias bibliográficas}

Boekema, F., Morgan, K., Bakkers, S. And Rutten, R. (2000). Knowledge, Innovation and Economic Growth: The Theory and Practice of Learning Regions, Edward Elgar Publishing Inc., Cheltenham/Northampton, M.A.

Casas, R. (Coord.) La formación de redes de conocimientos. Una perspectiva regional desde México, Edición Anthropos, IISUNAN, 2001, México.

Chi, M. T. H., Slotta, J. D., \& Leeuw, N. (1994). From things to processes: a theory of conceptual change for learning science concepts. Learning and instruction, 4, 27-43.

Cooke, P. A\& Leydesdorff, L. (2006). Journal of Technology Transfer, 31: 5-15. 2005. Springer Science Business Media, Inc. Manufactured in The Netherlands.

Fliaster, A. \& Spiess, J. (2008). Knowledge mobilization through social ties: The CostBenefit Analysis , Transaction Costs.100-102.

Garrat, B. (1990). Creating a knowledge organization: a guide to leadership. Learning \& Development. Cambridge, England: Director Books.

Holland, J. H. (1995). Hidden order: How adaptation builds complexity. New York: Helix Books.

Jonassen, D. \& Henning, P. (1996). Mental models in the head and knowledge in the world. International Conference on Learning Science. Proceedings of the 1996 Conference on Learning Science.

Leydesdorff, L. (1994). 'Epilogue,' in L. Leydesdorff and P.v.d. Besselaar (eds.), Evolutionary economics and Chaos Theory: New Directions for Technology studies, London, New York: Pinter, pp. 180-192. 
(2006). 'While a storm is raging on the open sea: Regional development in a knowledge- based economy. Journal of technology Transfer, 31: 189-203. Springer Science Business Media, Inc. Manufactured in The Netherlands.

LIEBOWITZ, J. (2005). Linking social network analysis with the analytic hierarchy process for knowledge mapping in organizations. Journal of knowledge management; 2005; 9, 1; BI/INFORM Global, p. 76.

Mansell, R. (2002). Constructing the knowledge base for knowledge-driven development. Journal of knowledge management; 6, 4; ABI/INFORM Global pp. 317

Martindale, M. J. (1993). Mental models and text schemes. Why computers based tutorials should be considered a communication medium. Journal of computer based instruction, 20(4), 107, 107-112.

Mischen, P. A \& Jackson, S. K. (2008). Connecting the dots: applying complexity theory, knowledge management and social network analysis to policy implementation. PAQ Fall, pp. 314-338, Binghamton University.

Morales G., I. (2007). Regional development through knowledge creation in organic agriculture. Journal of knowledge management, vol. 11 no. 5 2007, pp. 87-97.

Morgan, K. (1997). The Learning region: Institutions, innovation and regional renewal. Regional studies, Vol. 31.5, pp. 491- 503. Department of city and regional planning, University of Wales Cardiff, PO Box 906, Cardiff CF1 3YN, UK.

Nonaka, I. \& Takeuchi, H. (1995). The knowledge creating company: How the Japanese companies create the dynamics of innovation. Oxford, New York: Oxford University Press.

Rodríguez, A., Araújo, A. \& Yulianov, E. (2003). Redes virtuales para la gestión del conocimiento: El caso de las universidades. Revista del Centro para la gestión del conocimiento en la universidad, Universidad del País Vasco, 427-439.

Rohr, M. \& Reimann, P. (1998). Reasoning with multiple representations when acquiring the particulate model or matter. In Van Someren, M., Reimann, P., Boshuizen, H.P.A., and de Jong, T.: Learning with multiple representations. Oxford, UK: Pergamon Edit.

Royero, J.(2008). Las redes sociales de conocimiento: El nuevo reto de las organizaciones de investigación científica y tecnológica. Revista iberoamericana de educación.

Saaty, T. (1980). The Analytic hierarchy process. New York, N.: Mac Graw Hill. 
Scheel, C. (2002). Knowledge clusters of technological innovation systems. Journal of knowledge management, 6, 4; ABI/INFORM Global pp. 356-367.

Senge, P. (2000). The Leader's new work: Building learning organizations. In Morey, D. Maybury, M., and Thuraisinngham, B.(Editors): Knowledge management: Classic and contemporary works. Cambridge: The MIT Press. Reprinted from Sloan Management Review, 32 No 1, Fall (1990).

Stehr, N. (1999). The future of social inequality. Society; 36, 5; Research library core pp. 54 .

Wiig, K. M. (1994). Learning organization in the knowledge society: Practical perspectives on knowledge and knowledge transfer. Proceedings of the Thirty Five International ADCIS Conference, Nashville, Tennessee, February 15-19. 